\title{
UMA ANÁLISE DOS TRABALHOS APRESENTADOS NO II ENCONTRO LUSO-BRASILEIRO DE HISTÓRIA DA MATEMÁTICA SOBRE A VINDA DE MATEMÁTICOS PORTUGUESES PARA O BRASIL
}

\section{AN ANALYSIS OF THE PAPERS PRESENTED AT THE II LUSO-BRAZILIAN MEETING OF HISTORY OF MATHEMATICS ABOUT THE COMING OF PORTUGUESE MATHEMATICIANS TO BRAZIL}

\author{
Antonio Peixoto de Araujo Neto ${ }^{1}$ \\ Universidade Estadual de Maringá - UEM \\ Lucieli M. Trivizoli ${ }^{2}$ \\ Universidade Estadual de Maringá - UEM
}

\begin{abstract}
Resumo
Este trabalho é parte dos estudos desenvolvidos em uma pesquisa de doutorado iniciada em março de 2017 e que se encontra, portanto, em fase inicial. Na pesquisa de doutorado temos por objetivo investigar, historicamente, aspectos profissionais e a produção acadêmica do matemático português João Remy Teixeira Freire e a sua contribuição para a Educação Matemática no Brasil. Para este trabalho, temos por objetivo compreender, a partir dos trabalhos apresentados no II Encontro LusoBrasileiro de História da Matemática, aspectos da vinda de matemáticos portugueses para o Brasil. Este é o principal evento luso-brasileiro da área pois congrega matemáticos do Brasil e de Portugal e promove a discussão de temas relevantes para o desenvolvimento da ciência nesses países. Para a execução desta pesquisa, nos valemos da perspectiva metodológica da pesquisa bibliográfica. Após a escolha dos anais do II Encontro Luso-Brasileiro de História da Matemática, fizemos uma análise exploratória, pelo sumário, de todos os trabalhos apresentados no evento. Selecionamos cinco trabalhos cujos temas versam sobre a vinda de matemáticos portugueses para o Brasil, objetivo deste trabalho. Após a seleção dos trabalhos, realizamos a leitura seletiva de cada um deles, concentrando nossas análises para extrair dos textos informações sobre a vinda dos matemáticos para o Brasil. As nossas análises evidenciaram que o principal motivo da vinda dos matemáticos portugueses para o Brasil foi a expulsão por parte do governo de Carlos Salazar. De acordo com as nossas análises, há indícios de que o professor Remy Freire, assim como outros matemáticos portugueses, viu no Brasil a possibilidade de continuar a desenvolver seus trabalhos pela proximidade da língua e a abertura por parte da comunidade matemática brasileira e que a vinda desses matemáticos fortaleceu o desenvolvimento da matemática no Brasil, em especial no Pernambuco, destino da maioria deles.
\end{abstract}

Palavras-chave: História da Matemática no Brasil; Matemáticos Portugueses; Encontro Luso-Brasileiro de História da Matemática; Remy Freire.

\footnotetext{
${ }^{1}$ netopeixotoaraujo@hotmail.com

2 lmtrivizoli@uem.br
} 


\begin{abstract}
This work is part of the studies developed in a doctoral research started in March of 2017 and in initial stage. In the doctoral research, we aim to investigate, historically, professional aspects and the academic production of the Portuguese mathematician João Remy Teixeira Freire and his contribution to Mathematical Education in Brazil. For this paper, we aim to understand the aspects related to the arrival of Portuguese mathematicians to Brazil from the analyses of the papers presented at the II LusoBrazilian Meeting of History of Mathematics. This is the main Luso-Brazilian event in the field because it brings together mathematicians from Brazil and Portugal and promotes the discussion of relevant topics to the development of science in these countries. For this paper, we use the methodological perspective of the bibliographic research. After the choice of the Procedings of the II Luso-Brazilian Meeting of History of Mathematics, we did an exploratory analysis, by the summary of all the papers presented at the event. We selected five papers whose subjects deal with the coming of Portuguese mathematicians to Brazil, the focus of our research. After the selection of the papers, we performed the selective reading of each of them, concentrating on identify the information about the coming of Portuguese mathematicians to Brazil. Our analysis showed that the main reason for the arrival of Portuguese mathematicians to Brazil was the expulsion by the government of Carlos Salazar. Also there are indications that Professor Remy Freire, as well as other Portuguese Mathematicians, saw in Brazil the possibility of continuing to develop his works due to the proximity of the language and the openness on the part of the Brazilian mathematical community and that the coming of these mathematicians strengthened the development Mathematics in Brazil, especially in the state of Pernambuco, the destination of most of them.
\end{abstract}

Keywords: History of Mathematics in Brazil; Portuguese Mathematicians; LusoBrazilian Meeting of History of Mathematics; Remy Freire.

\title{
Introdução
}

Este trabalho é parte dos estudos desenvolvidos em uma pesquisa de doutorado iniciada em março de 2017 pelo Programa de Pós-Graduação em Educação para a Ciência e a Matemática - PCM da Universidade Estadual de Maringá - UEM e que se encontra em fase inicial.

$\mathrm{Na}$ pesquisa de doutorado, temos por objetivo investigar, historicamente, aspectos profissionais e a produção acadêmica de João Remy Teixeira Freire (Remy Freire) e a sua contribuição para a Educação Matemática no Brasil.

De acordo com Rezende (2011), o professor Remy Freire nasceu em Lisboa, Portugal, em 1919. Formou-se em Ciências Econômicas e Financeiras pela Universidade de Lisboa, onde também obteve seu doutorado em Ciências Econômicas. Além disso, era doutor em Estatística pela Universidade de Paris. Foi assistente de Bento de Jesus Caraça que contribuiu para o desenvolvimento do ensino da Matemática 
em Portugal. Remy Freire veio de Portugal para o Brasil em 1952 e foi um dos idealizadores para a criação da Sociedade Paranaense de Matemática (SPM) e grande difusor da Matemática em Curitiba, onde ficou erradicado até 1959 quando foi para o Chile ocupar uma posição na Organização das Nações Unidas (ONU).

O presente trabalho tem o intuito de compreender aspectos da vinda de matemáticos portugueses para o Brasil, a partir dos trabalhos apresentados nos Anais do II Encontro Luso-Brasileiro de História da Matemática. Por ser um evento que congrega historiadores da Matemática do Brasil e de Portugal, acreditamos que a análise dos trabalhos apresentados possibilitará uma compreensão da dinâmica do contexto da vinda do professor Remy para o Brasil.

O Encontro Luso-Brasileiro de História da Matemática acontece em períodos alternados de 4 e 3 anos, sendo uma edição no Brasil e outra em Portugal. É promovido em conjunto pela Sociedade Brasileira de História da Matemática e a Sociedade Portuguesa de Matemática. O evento já teve 7 edições, a saber:

Quadro 1 - Encontros realizados

\begin{tabular}{|c|c|c|c|}
\hline Edição & Ano & Período & Local \\
\hline I & 1993 & 31 de Agosto a 3 de Setembro & Coimbra - Portugal \\
\hline II & 1997 & 23 a 26 de Março & Águas de São Pedro - Brasil \\
\hline III & 2000 & 7 a 12 de Fevereiro & Coimbra - Portugal \\
\hline IV & 2004 & 24 a 27 de Outubro & Rio Grande do Norte - Brasil \\
\hline V & 2007 & 03 a 07 de Outubro & Castelo Branco - Portugal \\
\hline VI & 2011 & 28 a 31 de Agosto & São João Del Rei - Brasil \\
\hline VII & 2014 & 15 a 19 de Outubro & Óbidos - Portugal \\
\hline
\end{tabular}

Fonte: Página $W e b$ do $8^{\circ}$ Encontro Luso-Brasileiro de História da Matemática ${ }^{3}$

A escolha pela segunda edição do evento se deu por um dos quatro objetivos do II Encontro: "Divulgar e discutir as contribuições de matemáticos portugueses para o desenvolvimento da Matemática no Brasil” (NOBRE, 1997, p. 5), que vem ao encontro do objetivo deste trabalho.

\footnotetext{
${ }^{3}$ Disponível em: http://www.elbhm.com/2017/09/historia-dos-encontro-luso-brasileiro.html. Acesso em 28 de dezembro de 2017.
} 
Assim, no que segue, apresentamos os procedimentos metodológicos utilizados nesta pesquisa e os critérios para seleção dos trabalhos. Em seguida, descrevemos as informações dos trabalhos selecionados que corroboram com o objetivo deste artigo. Finalizamos este texto com uma análise geral e concisa das informações obtidas a partir das leituras dos textos apresentados no evento e as convergências com as informações levantadas sobre o professor Remy Freire até o momento.

\section{Procedimentos}

Neste trabalho nos valemos da perspectiva metodológica da pesquisa bibliográfica. Para Nascimento (2012, p. 58), a pesquisa bibliográfica "Consiste no desenvolvimento de uma pesquisa teórica, baseada na apresentação do pensamento dos autores pesquisados, fazendo-se uma análise comparativa das teorias divulgadas e buscando-se, através da crítica, apresentar um trabalho inovador".

Como exposto na introdução, o intuito deste trabalho é analisar os enfrentamentos da vinda de matemáticos portugueses para o Brasil, em especial a vinda do professor Remy Freire, objeto de estudo do trabalho de doutoramento que encontrase em andamento. Nascimento (2012, p. 6), alega que "a leitura com fins de pesquisa para o desenvolvimento de trabalhos acadêmicos se caracteriza pela identificação de dados e informações adequados, estabelecendo-se entre eles as relações com o problema proposto de modo a se analisar sua consistência”.

São diversos os tipos de leitura existentes e utilizados no desenvolvimento da pesquisa bibliográfica. Neste trabalho, utilizamos a leitura seletiva que segundo Nascimento (2012, p. 7), “tem por objetivo aprofundar conhecimentos sobre os pontos que se deseja alcançar por intermédio da pesquisa em desenvolvimento. Os textos a serem lidos dizem respeito diretamente ao assunto de interesse da elaboração da pesquisa".

Desta forma, seguimos as etapas metodológicas propostas por Nascimento (2012). Inicialmente, após a escolha dos anais do II Encontro Luso-Brasileiro de História da Matemática, fizemos uma análise exploratória, pelo sumário, de todos os trabalhos apresentados no evento. Selecionamos cinco trabalhos cujos temas versam sobre a vinda de matemáticos portugueses para o Brasil, objetivo deste trabalho. 
Após a seleção dos trabalhos, realizamos a leitura seletiva de cada um deles, concentrando nossas análises para extrair dos textos informações sobre a vinda dos matemáticos para o Brasil.

Concentramos as nossas leituras seguindo um parâmetro (pontos) de análise. Os pontos utilizados para a composição da descrição dos trabalhos selecionados na próxima seção deste texto foram: contexto da vinda, principais influências e enfrentamentos, lotação institucional no Brasil e possíveis entrelaçamentos com a vinda do professor Remy Freire. Esses pontos foram utilizados como critérios para a condução das leituras dos trabalhos do II Encontro mas não foram seguidos de forma rídiga e obrigatória pois os textos não seguem uma linha uniforme de informações que versassem sobre a temática deste trabalho.

\title{
Descrição dos Trabalhos Apresentados
}

Segundo Nobre (1997, p. 5), na apresentação dos Anais do evento, o II Encontro Luso-Brasileiro de História da Matemática teve por objetivos:

Divulgar e discutir as contribuições de matemáticos portugueses para o desenvolvimento da Matemática no Brasil; Incentivar o intercâmbio entre pesquisadores que trabalham na área de História da Matemática em países de língua portuguesa; Divulgar e discutir as pesquisas realizadas em História da Matemática e/ou no âmbito das relações entre História, Epistemologia e Educação Matemática; Dar andamento e implementar a "Coleção Memórias Vivas”, com a produção de vídeos sobre a História da Matemática no Brasil.

O tema daquela edição do evento foi "A contribuição de Matemáticos Portugueses para o desenvolvimento da Matemática no Brasil”. Nobre (1997), salienta que a escolha deste tema se deve às contribuições que os matemáticos portugueses tiveram no desenvolvimento da Matemática no Brasil:

\begin{abstract}
A investigação histórica apenas iniciou-se. Há, no entanto, muito a ser investigado nesta área. Exemplos recentes dessa "contribuição" encontra-se na atuação dos matemáticos António Aniceto Monteiro, Zaluar Nunes, Remy Freire, Ruy Luis Gomes, Alfredo Pereira Gomes e José Morgado, que, além de terem trabalhado em instituições brasileiras e atuado em favor do crescimento da Matemática no Brasil, foram professores de eminentes matemáticos brasileiros da atualidade. (NOBRE, 1997. p. 6)
\end{abstract}

Observamos a referência que Nobre (1997) faz ao professor Remy Freire sobre as suas contribuições para o desenvolvimento da Matemática no Brasil e a importância 
de investigações históricas como a que nos propomos no nosso trabalho de doutoramento.

Nas seções a seguir, apresentamos uma descrição concisa dos trabalhos publicados nos anais do II Encontro que remetem à vinda de matemáticos portugueses para o Brasil, seguindo as categorias sobre o contexto da vinda, principais influências e enfrentamentos, lotação institucional no Brasil e possíveis entrelaçamentos com a vinda do professor Remy Freire.

\section{Contribuição dos Militares Portugueses para a Introdução da Cultura Matemática} no Brasil - Belchior Vieira (Lisboa - Portugal)

Vieira (1997) observa que, historicamente, é possível afirmar que o ensino das matemáticas surgiu no Brasil-Colônia, a partir da segunda metade do século XVII, através das aulas militares, como base da ciência da fortificação, que então integrava o modelo de ensino artilharia-engenharia. Estas aulas foram, inicialmente, criadas em unidades de artilharia da organização militar que o Reino fizera implantar no Brasil com o regimento de D. João III (p. 45).

O autor diz que a necessidade de replicar no Brasil o modelo português de ensino militar, esteve ligada ao desejo de se desenvolver o conhecimento técnico na área de engenharia para construir núcleos de vida como base de uma estratégia de fomento e a inexistência de um ensino de nível superior no Brasil, que era propositadamente retardado pelo governo português pelo receio de que o desenvolvimento cultural no Brasil pudesse acelerar a emancipação.

\section{Implantação no Recife de um Núcleo De Matemáticos Portugueses na Década de} 50 - Alfredo Pereira Gomes (Universidade de Lusíada - Lisboa - Portugal)

Gomes (1997) observa que em 1950, recebeu em Paris a visita do professor de Matemática Newton Maia, da Universidade Federal de Pernambuco - UFPE por recomendação do professor Leopoldo Nachbin para iniciar e orientar um Departamento de Matemática na UFPE.

O objetivo, segundo Gomes (1997), era contratar dois matemáticos e um físico, qualificados e de preferência de língua portuguesa. A cadeira de físico foi ocupada por um brasileiro e as duas cadeiras da Matemática foram ocupadas pelos professores 
Alfredo Pereira Gomes e Manuel Zaluar Nunes, indicado pelo professor Alfredo Pereira Gomes. Os professores portugueses chegaram no Brasil em 1953.

Após alguns anos no Brasil, em 1957, o professor Alfredo Gomes convidou o seu amigo português José Morgado, que assim como outros professores portugueses foi afastado autoritariamente das funções universitárias que vinha exercendo. Após o aceite do convite pelo professor Morgado, outros professores portugueses também vieram por intermédio do professor Alfredo Gomes, a saber: Ruy Luis Gomes (1962) e António Brotas (1963).

O Professor Ruy Luis Gomes e o Núcleo Matemático do Recife (José Morgado (Universidade do Porto - Portugal)

O professor Ruy Luis Gomes, segundo Morgado (1997), veio para o Recife em 1962 para trabalhar no então chamado Instituto de Física e Matemática que mais tarde se desdobrou em dois Institutos - o Instituto de Física e o Instituto de Matemática. Assim como outros professores, Ruy Luis Gomes foi expulso do ensino universitário português pela ditadura salazarista.

Aluno de Ruy Luis Gomes, José Morgado observa:

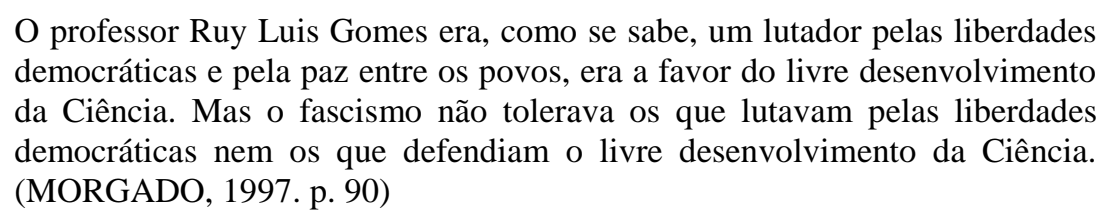

Desta forma, observamos a convergência do perfil dos matemáticos portugueses que vieram para o Brasil: expulsos do ensino superior e do exercício de ensino e de pesquisa pelo governo de Carlos Salazar. Segundo Morgado (1997), mais de vinte docentes vieram trabalhar para a Universidade do Recife.

António Aniceto Ribeiro Monteiro (1907 - 1980) no Brasil - Circe Mary Silva da Silva (UFES - Vitória)

Assim como os outros matemáticos portugueses, António Aniceto Ribeiro Monteiro saiu de Portugal por suas convicções políticas serem contrárias ao governo salazarista. Segundo Silva (1997), o professor António Monteiro chegou ao Brasil em 
1945 e iniciou as suas atividades docentes na Faculdade Nacional de Filosofia da Universidade do Brasil, no Rio de Janeiro.

Silva (1997) observa que o professor António Monteiro também ingressou como pesquisador do Núcleo Técnico Científico de Matemática da Fundação Getúlio Vargas, criado em 1945. Também escreveu um de seus importantes trabalhos no Brasil, intitulado "Filtros e Ideais I", publicado nas Notas de Matemática, em 1948.

António Monteiro, segundo Silva (1997), ficou no Brasil até 1949 e durante esse tempo marcou sua presença com atividades importantes influenciando um significativo número de futuros matemáticos no Brasil como, por exemplo, Leopoldo Nachbin. Após sua estada no Brasil, o professor António Monteiro foi para a Argentina.

António A. R. Monteiro - Um Matemático Português no Brasil - Elza Maria Alves de Sousa Amaral (UTAD - Portugal)

Segundo Amaral (1997), por falta de uma tradição de trabalho em Matemática em seu tempo, nenhum matemático da época de António Monteiro conseguiu provocar a efervescência matemática comparável ele. O autor chega a comparar essa situação como a de Pedro Nunes no século XIX, Anastácio da Cunha e Monteiro da Rocha no século XVIII, Gomes Teixeira no século XIX e Mira Fernandes e Vicente Gonçalves no início do período da República.

António Aniceto Ribeiro Monteiro, descreve Amaral (1997), nasceu em 31 de maio de 1907 em Mossâmedes (Angola). Foi licenciado em Ciências Matemáticas, em 1930, pela Faculdade de Ciências de Lisboa e o grau de doutor pela Universidade de Paris em 1936:

Terminado o doutoramento, em 1936, A. Monteiro regressa a Portugal (Lisboa) e, juntamente com Bento de Jesus Caraça, M. Zaluar Nunes, Manuel Valadares, Aurélio Marques da Silva, António da Silveira, e outros, funda o Núcleo de Matemática, Física e Química cujo principal objetivo era promover a realização de cursos e conferências nestas ciências. (AMARAL, 1997. p. 124)

Monteiro foi um dos responsáveis pela criação da Sociedade Portuguesa de Matemática e do Centro de Estudos Matemáticos de Lisboa. Entretanto, segundo Amaral (1997), o governo português colocava os mais variados obstáculos com o intuito 
de dificultar ou até mesmo impedir a enorme agitação científica que começava a despertar em Portugal.

\title{
Discussões
}

Vieira (1997) observa a fragilidade do ensino de nível superior no Brasil, que até o início do século XIX era propositadamente retardado pelo governo português pelo receio de que o desenvolvimento cultural no Brasil pudesse acelerar a emancipação.

Destacamos, de acordo com o exposto por Gomes (1997), os esforços mobilizados pelos professores Newton Maia e Leopoldo Nachbin, da Universidade Federal de Pernambuco - UFPE para iniciar e orientar um Departamento de Matemática na UFPE.

Segundo Morgado (1997), mais de vinte docentes portugueses vieram para o Brasil trabalhar na Universidade do Recife, conforme é confirmado por Amaral (1997):

\begin{abstract}
Nos anos de 1946 e 1947, o regime Salazarista desencadeou uma ofensiva contra a Universidade Portuguesa. Por diversos processos, foram afastados do ensino universitário (do Porto, de Lisboa e de Coimbra) ou impedidos de nele entrarem, entre outros: Bento Caraça, Mário de Azevedo Gomes, Ruy Luís Gomes, Pulido Valente, Fernando Fonseca, Ferreira de Macedo, Peres de Carvalho, Dias Amado, Celestino da Costa, Cândido de Oliveira, Adelino da Costa, Cascão de Anciães, Mário Silva, Torre de Assunção, Flávio Resende, Zaluar Nunes, Remy Freire, Crabée Rocha, Manuel Valadares, Armando Gibert, Lopes Raimundo, Laureano Barros, José Morgado, Morbey Rodrigues, Alfredo Pereira Gomes, Augusto Sá da Costa, Virgílio Barroso, Jorge Delgado, Hugo Ribeiro, António Monteiro, Fernando Soares David, Marques da Silva, António Santos Soares. (AMARAL, 1997. p. 127)
\end{abstract}

Dentre os matemáticos listados acima, Silva (1997) e Amaral (1997) destacam o professor português António Aniceto Ribeiro Monteiro, um dos mais renomados da sua época e responsável por marcos do desenvolvimento da Matemática em Portugal e no Brasil.

Observamos o quanto foi afetado o desenvolvimento científico matemático em Portugal com a expulsão da comunidade matemática da época pelo governo salazarista. De acordo com as nossas análises, há indícios de que o professor Remy Freire, assim como outros matemáticos, viu no Brasil a possibilidade de continuar a desenvolver seus trabalhos pela proximidade da língua e a abertura por parte da comunidade matemática brasileira. 
Segundo Nobre (1997), o professor Remy e os outros matemáticos portugueses, além de terem trabalhado em instituições brasileiras e atuado em favor do crescimento da Matemática no Brasil, deixaram como continuadores dos seus trabalhos 'discípulos' matemáticos que prosseguiram e disseminaram os estudos em outras Instituições pelo país.

\section{Considerações Finais}

O desenvolvimento científico de um país está fortemente ligado ao incentivo por parte dos governantes. Com a leitura dos trabalhos apresentados nos anais do II Encontro Luso-Brasileiro de História da Matemática, observamos o quão desastroso pode se tornar o progresso da ciência sem o apoio do governo.

Apesar dos motivos da vinda dos diversos matemáticos portugueses para o Brasil estarem pautados na expulsão pelo governo salazarista, o desenvolvimento da Matemática brasileira, em especial a pernambucana, foi fortalecido com os matemáticos refugiados de Portugal. Curitiba foi o destino do professor Remy Freire que, assim como tantos outros matemáticos portugueses, viu nas terras brasileiras uma possibilidade de continuar os estudos científicos que foram impedidos em sua terra natal.

Sabemos que este trabalho retrata genericamente este importante momento histórico para o desenvolvimento científico matemático no Brasil. De toda forma, o objetivo de compreender, a partir dos trabalhos apresentados no II Encontro LusoBrasileiro de História da Matemática, aspectos da vinda de matemáticos portugueses para o Brasil foi atendido na medida em que apresentamos uma descrição dos fatos expostos nos trabalhos selecionados. Indicamos como continuidade desta pesquisa, uma análise das outras seis edições do Encontro para a composição de um mapeamento da comunidade matemática portuguesa no Brasil a partir dessa fonte bibliográfica.

\section{Referências}

AMARAL, Elza Maria Alves de Sousa. António A. R. Monteiro - Um Matemático Português no Brasil. In: Encontro Luso-Brasileiro de História da Matemática, 2 ${ }^{\circ}$ 1997, Águas de São Pedro - São Paulo. Anais - Actas. Águas de São Pedro - São Paulo: Comitê Brasileiro de História da Matemática, 1997. p. 123 - 133.

GOMES, Alfredo Pereira. Implantação no Recife de um núcleo de matemáticos portugueses na década de 50. In: Encontro Luso-Brasileiro de História da Matemática, 
2 , 1997, Águas de São Pedro - São Paulo. Anais - Actas. Águas de São Pedro - São Paulo: Comitê Brasileiro de História da Matemática, 1997. p. 67 - 84.

MORGADO, José. O professor Ruy Luis Gomes e o núcleo matemático do Recife. In: Encontro Luso-Brasileiro de História da Matemática, 2º 1997, Águas de São Pedro São Paulo. Anais - Actas. Águas de São Pedro - São Paulo: Comitê Brasileiro de História da Matemática, 1997. p. 85 - 94.

NASCIMENTO, Luiz Paulo do. Elaboração de Projetos de Pesquisa: Monografia, dissertação, tese e estudo de caso, com base em metodologia científica. São Paulo: Cengage Learning, 2012.

NOBRE, Sergio. A investigação científica em História da Matemática em Portugal e no Brasil: a caminho para sua consolidação como área acadêmica. In: Encontro LusoBrasileiro de História da Matemática, 2º 1997, Águas de São Pedro - São Paulo. Anais - Actas. Águas de São Pedro - São Paulo: Comitê Brasileiro de História da Matemática, 1997. p. 1 - 7.

REZENDE, Jorge. António Aniceto Monteiro. 2011. Blog da Internet. Disponível em: $<$ http://antonioanicetomonteiro.blogspot.com.br/2011/04/joao-remy-teixeira-freireremy-freire.html>. Acesso em: 16 ago. 2016.

SILVA, Circe Mary Silva da. António Aniceto Ribeiro Monteiro (1907 - 1980) no Brasil. In: Encontro Luso-Brasileiro de História da Matemática, 2º 1997, Águas de São Pedro - São Paulo. Anais - Actas. Águas de São Pedro - São Paulo: Comitê Brasileiro de História da Matemática, 1997. p. 113 - 121.

VIEIRA, Belchior. Contribuição dos militares portugueses para a introdução da cultura matemática no Brasil. In: Encontro Luso-Brasileiro de História da Matemática, 2º, 1997, Águas de São Pedro - São Paulo. Anais - Actas. Águas de São Pedro - São Paulo: Comitê Brasileiro de História da Matemática, 1997. p. 45 - 51. 